\title{
Additive rules in bankruptcy problems and other related problems*
}

\author{
Gustavo Bergantiños ${ }^{\dagger}$ \\ Research Group in Economic Analysis, \\ Facultade de Económicas, \\ Universidade de Vigo, \\ 36200 Vigo, Pontevedra, Spain. \\ Juan J. Vidal-Puga \\ Facultade de Económicas, \\ Universidade de Vigo, \\ 36200 Vigo, Pontevedra, Spain. \\ Published in January 2004 in Mathematical Social Sciences
}

\begin{abstract}
In this paper we characterize the set of rules satisfying additivity on the estate along with additivity on the estate and the claims in bankruptcy problems and other related problems. Moreover, new characterizations of the well known rules based on the principles of "equal award", "equal loss", and "proportionality" are provided using these additivity properties.
\end{abstract}

Keywords: Additivity; Bankruptcy; Proportional rule

JEL classification: C79

*The authors wish to thank Luciano Méndez-Naya and Carlos Hervés for their helpful comments. Financial support from the Ministerio de Ciencia y Tecnologia and FEDER (grants PB98-0613-C02-01 and BEC2002-04102-C02-01) and Xunta de Galicia (grant PGIDT00PXI30001PN) is gratefully acknowledged.

${ }^{\dagger}$ Corresponding author. Tel.: +34-986-812497; fax: +34-986-812401.

E-mail addresses: gbergant@uvigo.es (G. Bergantiños), vidalpuga@uvigo.es (J. Vidal-Puga).

¥DOI: 10.1016/S0165-4896(03)00079-9. Creative Commons Attribution Non-Commercial No Derivatives License. 


\section{Introduction}

Many economic situations can be modelled as a problem of how to divide a resource among agents who have claims on it. In this paper we study problems where an estate $E$ must be divided among a finite group of agents $N, c_{i}$ being the claim of agent $i$.

We study four kinds of problems that differ in the way an estate must be divided. In bankruptcy problems (introduced by O’Neill (1982) and studied later by Aumann and Maschler (1985)) an agent must receive at least 0 and at most his claim. In allocation problems (Chun (1988) and Herrero, Maschler, and Villar (1999)) agents can receive anything. In surplus problems (Moulin (1987)) every agent must receive at least 0. In loss problems, defined in this paper, every agent must receive at most his claim. Notice that with the four classes of problems we cover all the possibilities.

One of the most important topics of these problems is the axiomatic characterizations of rules. The idea is to propose desirable properties and find out which of them characterize every rule. Properties often help agents to compare different rules and to decide which rule is preferred for a particular situation. Thomson (2003) and Moulin (2002) give a survey of this literature.

A dual approach is to study what the rules satisfying a set of properties are. For instance, Young (1988) characterizes the rules satisfying continuity, symmetry, and consistency; de Frutos (1999) characterizes the rules satisfying non-manipulability; and Moulin (2000) characterizes the rules satisfying consistency, composition up, composition down, and scale invariance.

In this paper we adopt both approaches. We characterize the rules satisfying additivity in each of the four problems mentioned above. Moreover, using these additivity properties, we characterize the well-known rules based on the principles of "proportionality", "equal award", and "equal loss".

Additivity is a standard property. It has been used in many situations. Although the justification of additivity is not as clear as with other properties (for example, efficiency or symmetry), in most cases it produces very interesting classes of rules. For instance, the Shapley value, the most important value in cooperative games with transferable utility, is characterized by additivity and other properties. If we compare the Shapley value with other prominent values (for example the nucleolus) we realize that these values satisfy all the properties characterizing the Shapley value except additivity.

In this paper we use two definitions of additivity: additivity on the estate (Moulin (1987) and Chun (1988)), called $A 1$, and additivity on the estate and the claims (Bergantiños and Méndez-Naya (2001)), called $A 2$. In the four kinds of problems we characterize the 
rules satisfying $A 1$ and $A 2$.

The rules satisfying $A 1$ are as follows. In allocation problems they are characterized by the product of the estate and a claims-depending function. In surplus problems the estate is divided among agents according to a weight system, which depends on the claims. In loss and bankruptcy problems only the proportional rule satisfies $A 1$.

The rules satisfying $A 2$ are as follows. In allocation problems they are characterized by the sum of two parts: one depending on the estate and the other depending on the claims. In surplus (loss) problems the estate (loss) is divided among agents according to a weight system, independent of the claims. There is no bankruptcy rule satisfying $A 2$.

We obtain axiomatic characterizations of well-known rules. In allocation problems and surplus problems, the proportional rule is characterized by $A 1$ and other properties. In allocation and loss problems, the rights-egalitarian rule (Herrero et al. (1999)) is characterized by $A 2$ and other properties. Moreover, $A 2$ and other properties also characterize the equal-sharing rule (Moulin (1987)) in surplus problems.

As a consequence of our results we can say that additivity properties also support the use of rules based on the three classical principles. $A 1$ is related to the principle of "proportionality"; $A 2$ is related to the principles of "equal award" and "equal loss".

The paper is organized as follows. Section 2 introduces the problems studied in this paper. In Section 3 we characterize the rules satisfying $A 1$ and $A 2$. In Section 4 we characterize well-known rules using these additivity properties. Section 5 is devoted to concluding remarks.

\section{Preliminaries}

We introduce some notation. $\mathbb{Z}$ denotes the set of integer numbers and $\mathbb{N}$ denotes the set of non-negative integer numbers. $\mathbb{Q}$ denotes the set of rational numbers and $\mathbb{Q}_{+}$denotes the set of non-negative rational numbers. $\mathbb{R}$ denotes the set of real numbers and $\mathbb{R}_{+}$the set of non-negative real numbers.

$\mathbb{N}$ also denotes the set of potential agents. Let $N$ be any finite subset of $\mathbb{N}$. Given $x, y \in \mathbb{R}^{N}, x \geq y$ means $x_{i} \geq y_{i}$ for all $i \in N ; x+y=\left(x_{i}+y_{i}\right)_{i \in N}$. Moreover, $0_{N}=(0, \ldots, 0) \epsilon$ $\mathbb{R}^{N}$. Given $S \subset N, 1_{S}=\left(x_{i}\right)_{i \in N}$ such that $x_{i}=1$ if $i \in S$ and $x_{i}=0$ if $i \notin S$.

We study problems where an estate $E$ must be divided among a group of agents $N, c_{i}$ being the claim of agent $i \in N, c=\left(c_{i}\right)_{i \in N}$ the vector of claims, and $C=\sum_{i \in N} c_{i}$ the sum of the claims. We assume that the estate and the claims are non-negative real numbers, $i$. e. $(c, E) \in \mathbb{R}_{+}^{N} \times \mathbb{R}_{+}$. The question that arises is: how to divide the estate among agents? 
This question is answered by means of defining rules. A rule, $f$, is a map which assigns to any problem $(c, E)$ a vector $f(c, E)$ where $f_{i}(c, E)$ denotes the part of the estate received by agent $i \in N$.

We now give a list of problems that fit in our general framework. Notice that the difference among these problems is, mainly, in the definition of what a rule is.

A bankruptcy problem, $B P$, is a pair $(c, E) \in \mathbb{R}_{+}^{N} \times \mathbb{R}_{+}$where $C \geq E$. We denote by $\mathcal{B}$ the set of all bankruptcy problems. A bankruptcy rule is a function $f^{B}: \mathcal{B} \rightarrow \mathbb{R}^{N}$ satisfying that for all $(c, E) \in \mathcal{B}, \sum_{i \in N} f_{i}^{B}(c, E)=E$ and $0 \leq f_{i}^{B}(c, E) \leq c_{i}$ for all $i \in N$.

A surplus problem, $S P$, is a pair $(c, E) \in \mathbb{R}_{+}^{N} \times \mathbb{R}_{+}$. We denote by $\mathcal{S}$ the set of all surplus problems. A surplus rule is a function $f^{S}: \mathcal{S} \rightarrow \mathbb{R}^{N}$ satisfying that for all $(c, E) \in \mathcal{S}, \sum_{i \in N} f_{i}^{S}(c, E)=E$ and $0 \leq f_{i}^{S}(c, E)$ for all $i \in N$.

An allocation problem, $A P$, is a pair $(c, E) \in \mathbb{R}_{+}^{N} \times \mathbb{R}_{+}$. We denote by $\mathcal{A}$ the set of all allocation problems. An allocation rule is a function $f^{A}: \mathcal{A} \rightarrow \mathbb{R}^{N}$ satisfying that for all $(c, E) \in \mathcal{A}, \sum_{i \in N} f_{i}^{A}(c, E)=E$.

These problems have been studied in the literature. For instance, O'Neill (1982) and Aumann and Maschler (1985) studied bankruptcy problems, Moulin (1987) studied surplus problems, and Chun (1988) and Herrero et al. (1999) studied allocation problems 1 . See Thomson (2003) and Moulin (2002) for a survey of this literature.

As far as we know the next class of problems has not been studied explicitly. A loss problem, $L P$, is a pair $(c, E) \in \mathbb{R}_{+}^{N} \times \mathbb{R}_{+}$where $C \geq E$. We denote by $\mathcal{L}$ the set of all loss problems. A loss rule is a function $f^{L}: \mathcal{L} \rightarrow \mathbb{R}^{N}$ satisfying that for all $(c, E) \in \mathcal{L}$, $\sum_{i \in N} f_{i}^{L}(c, E)=E$ and $f_{i}^{L}(c, E) \leq c_{i}$ for all $i \in N$.

Remark 1. These four kinds of problems cover all possible definitions of a rule. Given $i \in N$, a bankruptcy rule satisfies $0 \leq f_{i}^{B}(c, E) \leq c_{i}$, a surplus rule satisfies $0 \leq f_{i}^{S}(c, E)$, a loss rule satisfies $f_{i}^{L}(c, E) \leq c_{i}$, and an allocation rule has no restrictions at all. Notice that any bankruptcy rule is a loss rule and any surplus rule is an allocation rule.

Herrero et al. (1999) consider the case where $E<0$. It is easy to check that our results are also valid if we allow the estate to be negative in $A P$ and $L P$.

In $B P$ and $L P$ we need to impose the condition $C \geq E$ because otherwise it is not possible to find $f$ satisfying $\sum_{i \in N} f_{i}(c, E)=E$ and $f_{i}(c, E) \leq c_{i}$ for all $i \in N$.

We now present some rules studied in this paper. The proportional rule, $P$, divides the estate proportionally to the claims when their sum $C$ is different from zero. Formally,

\footnotetext{
${ }^{1}$ Chun (1988) refers to allocation problems as rights problems.
} 
for all $i \in N, c \neq 0_{N}$,

$$
P_{i}(c, E)=\lambda c_{i} \text { where } \lambda=\frac{E}{C} .
$$

If $c=0_{N}$ then, for all $i \in N, P_{i}\left(0_{N}, E\right)=\frac{E}{n}$.

Remark 2. It is easy to check that the results obtained in this paper are also true if we define $P\left(0_{N}, E\right)$ in a different way.

Assume that $c=0_{N}$ and $(c, E)$ is a $B P$ or a $L P$. Since $C \geq E \geq 0$, we conclude that $E=0$. Then, any bankruptcy rule or loss rule satisfies $f\left(0_{N}, 0\right)=0_{N}$. Thus, $P\left(0_{N}, 0\right)=$ $0_{N}$.

The equal-sharing rule, ES, divides the estate equally among agents. Formally, for all $i \in N$,

$$
E S_{i}(c, E)=\frac{E}{n} .
$$

Notice that the equal-sharing rule is both an allocation rule and a surplus rule. Nevertheless, it is not a bankruptcy rule or a loss rule because $E S_{i}(c, E)$ could be larger than $c_{i}$.

We present a family of rules closely related to ES. We denote $\omega$ as a weight system if $\omega \in \mathbb{R}_{+}^{N}$ and $\sum_{i \in N} \omega_{i}=1$.

The weighted-sharing rule $W S^{\omega}$ with weight system $\omega$ is defined for all $i \in N$ as

$$
W S_{i}^{\omega}(c, E)=\omega_{i} E
$$

Notice that $W S^{\omega}$ is an allocation rule and a surplus rule but not a bankruptcy rule or a loss rule. Of course, if $\omega_{i}=\frac{1}{n}$ for all $i \in N$, then $W S^{\omega}=E S$.

Herrero et al. (1999) define the rights-egalitarian rule, $R E$, for all $i \in N$ as

$$
R E_{i}(c, E)=c_{i}-\frac{1}{n}(C-E) .
$$

Notice that the rights-egalitarian rule is an allocation rule and a loss rule (in $L P, C \geq E$ ). Nevertheless, it is not a bankruptcy rule or a surplus rule because $R E_{i}(c, E)$ could be negative.

We present other rules closely related to $R E$. The weighted-rights rule $W R^{\omega}$ with weight system $\omega$ is defined for all $i \in N$ as

$$
W R_{i}^{\omega}(c, E)=c_{i}-\omega_{i}(C-E)
$$

Notice that $W R^{\omega}$ is an allocation rule and a loss rule but not a bankruptcy rule or a surplus rule. Of course, if $\omega_{i}=\frac{1}{n}$ for all $i \in N$, then $W R^{\omega}=R E$. 
We now present some properties used in this paper. First, we introduce the two additivity properties considered in this paper.

Additivity on $E(A 1)$. For all $(c, E)$ and $\left(c, E^{\prime}\right)$,

$$
f\left(c, E+E^{\prime}\right)=f(c, E)+f\left(c, E^{\prime}\right) .
$$

Moulin (1987) and Chun (1988) used this property in surplus problems and in allocation problems, respectively. $A 1$ indicates that to divide the estate among the agents is the same as dividing, first, one part of the estate, and afterwards, the remaining estate.

Additivity on $(c, E)$ (A2). For all $(c, E)$ and $\left(c^{\prime}, E^{\prime}\right)$,

$$
f\left(c+c^{\prime}, E+E^{\prime}\right)=f(c, E)+f\left(c^{\prime}, E^{\prime}\right)
$$

Bergantiños and Méndez-Naya (2001) introduced this property in bankruptcy problems and in allocation problems. Suppose that the product sold by a firm depends on several parts (quality and marketing, for instance). The total revenue of the firm, $E+E^{\prime}$, can be divided into two parts: one motivated by quality $(E)$ and the other by marketing $\left(E^{\prime}\right)$. We can also determine the contribution of every agent of the firm to quality $(c)$ and marketing $\left(c^{\prime}\right)$. Now we can allocate the revenue according to two procedures. First, we allocate the total revenue $\left(E+E^{\prime}\right)$ according to the total contribution $\left(c+c^{\prime}\right)$. Second, we allocate the revenue motivated by quality $(E)$ according to the contribution to quality $(c)$, and the revenue of marketing $\left(E^{\prime}\right)$ according to the contribution to marketing $\left(c^{\prime}\right)$. $A 2$ guarantees that both procedures coincide.

Usually it is not very difficult to determine the contribution of the agents to each part (for instance, hours worked) and to the total revenue. But sometimes it seems impossible to know exactly the contribution of each part to the total revenue. Under these circumstances it appears that the second procedure cannot be applied. However, if the allocation rule satisfies $A 2$, its application is possible since both procedures coincide.

There is no relation between $A 1$ and $A 2$. Later, we will see examples of rules satisfying $A 1$ but not $A 2$ and rules satisfying $A 2$ but not $A 1$.

More properties will now be considered. Symmetry and continuity are standard properties that could be defined in each of the four problems studied in this paper.

Symmetry (SYM). For all problems $(c, E)$, if $c_{i}=c_{j}$, then $f_{i}(c, E)=f_{j}(c, E)$.

Continuity on $E(C O N T)$. For all sequences of problems $\left(c, E^{l}\right)$ and all problems $(c, E)$, if $E^{l} \rightarrow E$, then $f\left(c, E^{l}\right) \rightarrow f(c, E)$.

Suppose that the estate, $E$, is equal to the sum of the claims, $C$. Compatibility denotes that each agent gets exactly the amount he claims. 
Compatibility (COM). For all problems $(c, C), f(c, C)=c$.

The next property appeared in a preliminary draft of Herrero et al. (1999), but not in the final version. Condition i) says that no agent gets less than he has a right to. Condition ii) says that no agent gets more than he claims.

Claims Boundedness $(C B)$. For all $(c, E) \in \mathcal{A}$ :

i) $f^{A}(c, E) \geq c$ if $C \leq E$.

ii) $f^{A}(c, E) \leq c$ if $C \geq E$.

Notice that all bankruptcy rules and loss rules satisfy $C B$.

\section{Additive rules}

In this section we characterize the set of additive rules in the four problems. In Theorem 1 we characterize the rules satisfying $A 1$ and in Theorem 2 the rules satisfying $A 2$.

Theorem 1. a) An allocation rule $f^{A}$ satisfies $A 1$ and $C O N T$ if and only if for all $(c, E) \in \mathcal{A}$,

$$
f_{i}^{A}(c, E)=E \alpha_{i}(c) \text { for all } i \in N
$$

where $\alpha: \mathbb{R}_{+}^{N} \rightarrow \mathbb{R}^{N}$ satisfies $\sum_{i \in N} \alpha_{i}(c)=1$ for all $c \in \mathbb{R}_{+}^{N}$.

b) A surplus rule $f^{S}$ satisfies $A 1$ if and only if for all $(c, E) \in \mathcal{S}$,

$$
f_{i}^{S}(c, E)=E \omega_{i}(c) \text { for all } i \in N
$$

where $\omega: \mathbb{R}_{+}^{N} \rightarrow \mathbb{R}_{+}^{N}$ is such that $\omega(c)$ is a weight system for every $c \in \mathbb{R}_{+}^{N}$.

c) The proportional rule is the only loss rule satisfying $A 1$.

d) The proportional rule is the only bankruptcy rule satisfying $A 1$.

Proof. a) It is trivial to prove that any function given by $f^{A}(c, E)=E \alpha(c)$ satisfies $A 1$ and $C O N T$.

We now prove the converse. Assume that $f^{A}$ is an allocation rule satisfying $A 1$ and $C O N T$. Since $f^{A}$ satisfies $A 1$ we conclude that:

- $f^{A}(c, E)=E f^{A}(c, 1)$ for all $E \in \mathbb{N}$.

- If $E=\frac{1}{q}$, where $q \in \mathbb{N}$, then $f^{A}(c, E)=E f^{A}(c, 1)$.

- Given $E \in \mathbb{Q}_{+}, f^{A}(c, E)=E f^{A}(c, 1)$. 
Since $f^{A}$ satisfies $C O N T, f^{A}(c, E)=E f^{A}(c, 1)$ for all $E \in \mathbb{R}_{+}$.

If we take $\alpha(c)=f^{A}(c, 1)$ the result holds.

b) It is trivial to prove that any function given by $f^{S}(c, E)=E \omega(c)$ satisfies $A 1$.

We now prove the converse. Assume that $f^{S}$ is a surplus rule satisfying $A 1$. Using arguments similar to those used in part a) we can conclude that given $E \in \mathbb{Q}_{+}, f^{S}(c, E)=$ $\operatorname{Ef} S(c, 1)$.

Let $E$ be a non-negative irrational number $\left(E \in \mathbb{R}_{+} \backslash \mathbb{Q}_{+}\right)$. Then, there exists $\left(E^{l}\right)_{l \in \mathbb{N}}$ such that $E^{l} \in \mathbb{Q}_{+}, 0<E^{l}<E$, and $\lim _{l \rightarrow \infty} E^{l}=E$. Thus, for all $l \in \mathbb{N}$,

$$
f^{S}\left(c, E-E^{l}\right)=f^{S}(c, E)-f^{S}\left(c, E^{l}\right)=f^{S}(c, E)-E^{l} f^{S}(c, 1) .
$$

Since $f^{S}\left(c, E-E^{l}\right) \geq 0_{N}$ and $\sum_{i \in N} f_{i}^{S}\left(c, E-E^{l}\right)=E-E^{l}$, for all $i \in N$,

$$
f_{i}^{S}\left(c, E-E^{l}\right) \leq E-E^{l} .
$$

Thus, for all $i \in N$,

$$
0 \leq \lim _{l \rightarrow \infty} f_{i}^{S}\left(c, E-E^{l}\right) \leq E-\lim _{l \rightarrow \infty} E^{l}=0
$$

So, for all $i \in N$,

$$
0=\lim _{l \rightarrow \infty} f_{i}^{S}\left(c, E-E^{l}\right)=f_{i}^{S}(c, E)-E f_{i}^{S}(c, 1) .
$$

Hence, $f^{S}(c, E)=E f^{S}(c, 1)$ for all $(c, E) \in \mathcal{S}$.

If we take $\omega(c)=f^{S}(c, 1)$ the result holds.

c) It is trivial to prove that the proportional rule satisfies $A 1$.

We now prove the converse. Assume that $f^{L}$ is a loss rule satisfying $A 1$. We first prove that $f^{L}(c, \varepsilon E)=E f^{L}(c, \varepsilon)$ when $(c, \varepsilon E) \in \mathcal{L},(c, \varepsilon) \in \mathcal{L}, E \in \mathbb{R}_{+}$, and $\varepsilon \in \mathbb{Q}_{+}$.

Using arguments similar to those used in part a) we can conclude that, given $c \in$ $\mathbb{R}_{+}^{N}$ such that $C>0,(c, \varepsilon E) \in \mathcal{L},(c, \varepsilon) \in \mathcal{L}, E \in \mathbb{Q}_{+}$, and $\varepsilon \in \mathbb{Q}_{+}$,

$$
f^{L}(c, \varepsilon E)=E f^{L}(c, \varepsilon) .
$$

Given $(c, \varepsilon E) \in \mathcal{L}$ with $E$ a non-negative irrational number, there exists $\left(E^{l}\right)_{l \in \mathbb{N}}$ such that $E^{l} \in \mathbb{Q}_{+}, 0<E^{l}<E$, and $\lim _{l \rightarrow \infty} E^{l}=E$. Thus, for all $l \in \mathbb{N}$,

$$
f^{L}\left(c, C-\left(\varepsilon E-\varepsilon E^{l}\right)\right)=c-f^{L}(c, \varepsilon E)+E^{l} f^{L}(c, \varepsilon) .
$$

Since $f^{L}\left(c, C-\left(\varepsilon E-\varepsilon E^{l}\right)\right) \leq c$ and $\sum_{i \in N} f_{i}^{L}\left(c, C-\left(\varepsilon E-\varepsilon E^{l}\right)\right)=C-\left(\varepsilon E-\varepsilon E^{l}\right)$, for all $i \in N$,

$$
f_{i}^{L}\left(c, C-\left(\varepsilon E-\varepsilon E^{l}\right)\right) \geq c_{i}-\left(\varepsilon E-\varepsilon E^{l}\right)
$$


Thus, for all $l \in \mathbb{N}$,

$$
c-\left(\varepsilon E-\varepsilon E^{l}\right) 1_{N} \leq f^{L}\left(c, C-\left(\varepsilon E-\varepsilon E^{l}\right)\right) \leq c .
$$

So, $c=\lim _{l \rightarrow \infty} f^{L}\left(c, C-\left(\varepsilon E-\varepsilon E^{l}\right)\right)=c-f^{L}(c, \varepsilon E)+E f^{L}(c, \varepsilon)$. Hence, $f^{L}(c, \varepsilon E)=$ $E f^{L}(c, \varepsilon)$ for all $E \in \mathbb{R}_{+}$such that $(c, \varepsilon E) \in \mathcal{L}$ and $(c, \varepsilon) \in \mathcal{L}$.

We now prove that $f^{L}$ is the proportional rule.

If $c=0_{N}$ and $(c, E) \in \mathcal{L}$, then $E=0$ and $f^{L}\left(0_{N}, 0\right)=0_{N}$.

Assume that $(c, E) \in \mathcal{L}$ and $c \neq 0_{N}$. Let $\varepsilon \in \mathbb{Q}_{+}$be such that $C>\varepsilon$. Then,

$$
f^{L}(c, E)=f^{L}\left(c, \frac{E}{\varepsilon} \varepsilon\right)=\frac{E}{\varepsilon} f^{L}(c, \varepsilon) .
$$

We know that,

$$
c=f^{L}\left(c, \frac{C}{\varepsilon} \varepsilon\right)=\frac{C}{\varepsilon} f^{L}(c, \varepsilon)
$$

which implies that $f^{L}(c, \varepsilon)=\frac{\varepsilon}{C} c$. Then,

$$
f^{L}(c, E)=\frac{E}{\varepsilon} \frac{\varepsilon}{C} c=\frac{E}{C} c=P(c, E) .
$$

d) Since every bankruptcy rule is a loss rule, part d) is an immediate consequence of c).

Remark 3. For $n>1$, condition $C O N T$ in a) is needed to avoid an infinite family of meaningless solutions. Let $\left(B^{l}\right)_{l \in \mathbb{L}}$ be a Hamel basis of $\mathbb{R}$ as vector space over $\mathbb{Q}$ with $B^{l}>0$ for all $l \in \mathbb{L}$ (see, for instance, Aczél and Dhombres (1989) for a detailed explanation). Let $\gamma: \mathbb{R}_{+}^{N} \times \mathbb{L} \rightarrow \mathbb{R}^{N}$ be any function satisfying

$$
\sum_{i \in N} \gamma_{i}(c, l)=B^{l}
$$

for all $(c, l) \in \mathbb{R}_{+}^{N} \times \mathbb{L}$.

We define $f^{A}$ as follows. Given $E \in \mathbb{R}_{+}$, there exists a unique $\left\{q^{1}, \ldots, q^{m}\right\} \subset \mathbb{Q}$ and $\left\{B^{l_{1}}, \ldots, B^{l_{m}}\right\}$ such that $E=\sum_{k=1}^{m} q^{k} B^{l_{k}}$. Thus,

$$
f^{A}(c, E)=\sum_{k=1}^{m} q^{k} \gamma\left(c, l_{k}\right)
$$

is well-defined, it satisfies $A 1$, and $\sum_{i \in N} f_{i}^{A}(c, E)=E$ for all $E \in \mathbb{R}_{+}$. However, by choosing an appropriate function $\gamma$, it is not of the form $f^{A}(c, E)=E \alpha(c)$. For example, given $l_{0} \in \mathbb{L}$, we define $\gamma$ as follows: 


$$
\gamma(c, l)= \begin{cases}1_{\{1\}} B^{l_{0}} & \text { if } l=l_{0} \\ 1_{N} \frac{B^{l}}{n} & \text { otherwise. }\end{cases}
$$

The associated function $f^{A}$ is an allocation rule satisfying $A 1$. However, it cannot be written as $f^{A}(c, E)=E \alpha(c)$.

Chun (1988) characterizes, in Theorem 4, the class of allocation rules satisfying $A 1$, continuity on the estate and the claims $\left(C O N T^{*}\right), S Y M^{2}$ and Pareto optimality. Notice that in our paper Pareto optimality is already included in the definition of an allocation rule.

Assume that we restrict ourselves to allocation problems where $C>0$, as Chun (1988) does. Using arguments similar to those used in the proof of Theorem 1, part a), we can conclude the following. An allocation rule satisfies $A 1, C O N T^{*}$, and $S Y M$ if and only if for all $(c, E) \in \mathcal{A}$,

$$
f^{A}(c, E)=E \alpha(c)
$$

where $\alpha: \mathbb{R}_{+}^{N} \rightarrow \mathbb{R}^{N}$ is a continuous function satisfying $\sum_{i \in N} \alpha_{i}(c)=1$ for all $c \in \mathbb{R}_{+}^{N}$ and $\alpha_{i}(c)=\alpha_{j}(c)$ whenever $c_{i}=c_{j}$.

Of course, these rules coincide with the class of rules characterized in Theorem 4 of Chun (1988), although the formulation is different.

It is straightforward to prove that an allocation rule $f^{A}$ satisfies $A 1, C O N T$, and $S Y M$ if and only if for all $(c, E) \in \mathcal{A}, f^{A}(c, E)=E \alpha(c)$ where $\alpha: \mathbb{R}_{+}^{N} \rightarrow \mathbb{R}^{N}$ satisfies $\sum_{i \in N} \alpha_{i}(c)=1$ for all $c \in \mathbb{R}_{+}^{N}$ and $\alpha_{i}(c)=\alpha_{j}(c)$ whenever $c_{i}=c_{j}$.

Notice that in part d) we obtain a new characterization of the proportional bankruptcy rule. Also, the proportional loss rule can be characterized as the only loss rule satisfying $A 1$.

The next theorem characterizes the rules satisfying $A 2$.

Theorem 2. a) An allocation rule $f^{A}$ satisfies $A 2$ and $C O N T$ if and only if for all $(c, E) \in \mathcal{A}$,

$$
f_{i}^{A}(c, E)=\beta_{i}(c)+E x_{i} \text { for all } i \in N,
$$

where $\beta: \mathbb{R}_{+}^{N} \rightarrow \mathbb{R}^{N}$ is a function satisfying $\sum_{i \in N} \beta_{i}(c)=0$ for all $c \in \mathbb{R}_{+}^{N}$ and $\beta\left(c+c^{\prime}\right)=$ $\beta(c)+\beta\left(c^{\prime}\right)$ for all $c, c^{\prime} \in \mathbb{R}_{+}^{N}$. Moreover, $x \in \mathbb{R}^{N}$ and $\sum_{i \in N} x_{i}=1$.

\footnotetext{
${ }^{2}$ Chun (1988) uses anonymity instead of symmetry. Nevertheless, our comments are also valid if we write anonymity instead of symmetry.
} 
b) A surplus rule $f^{S}$ satisfies $A 2$ if and only if it is a weighted-sharing rule $W S^{\omega}$ for some weight system $\omega$.

c) A loss rule $f^{L}$ satisfies $A 2$ if and only if it is a weighted-rights rule $W R^{\omega}$ for some weight system $\omega$.

d) There is no bankruptcy rule satisfying $A 2$.

Proof. a) It is trivial to prove that if $f^{A}(c, E)=\beta(c)+E x$ then $f^{A}$ satisfies $A 2$ and CONT.

We now prove the converse. Since $f^{A}$ satisfies $A 2$ we conclude that $f^{A}(c, E)=$ $f^{A}\left(0_{N}, E\right)+f^{A}(c, 0)$.

Using arguments similar to those used in the proof of Theorem 1 a) we can conclude that $f^{A}\left(0_{N}, E\right)=E f^{A}\left(0_{N}, 1\right)$ for all $E \in \mathbb{R}_{+}$.

If we define $\beta(c)=f^{A}(c, 0)$ and $x=f^{A}\left(0_{N}, 1\right)$, the result holds.

b) It is trivial to prove that every weighted-sharing rule $W S^{\omega}$ satisfies $A 2$.

We now prove the converse. Assume that $f^{S}$ is a surplus rule satisfying $A 2$.

Using arguments similar to those used in the proof of Theorem $1 \mathrm{~b}$ ) we can conclude that given $E \in \mathbb{R}_{+}, f^{S}\left(0_{N}, E\right)=E f^{S}\left(0_{N}, 1\right)$.

If we define $\beta(c)=f^{S}(c, 0)$ and $x=f^{S}\left(0_{N}, 1\right)$, every surplus rule has the form given in part a). Since $f^{S}(c, E) \geq 0_{N}$ for all $(c, E) \in \mathcal{S}, \beta(c)=f^{S}(c, 0) \geq 0_{N}$. As $\sum_{i \in N} f_{i}^{S}(c, 0)=0$ we conclude that $f^{S}(c, 0)=0_{N}$. Now it is easy to prove that $f^{S}=W S^{\omega}$ where $\omega=f^{S}\left(0_{N}, 1\right)$ is a weight system.

c) It is trivial to prove that every weighted-rights rule $W R^{\omega}$ satisfies $A 2$.

We now prove the converse. Assume that $f^{L}$ is a loss rule satisfying $A 2$.

We first prove that for all $i \in N$ and $x \in \mathbb{R}_{+}, f^{L}\left(x 1_{\{i\}}, 0\right)=x f^{L}\left(1_{\{i\}}, 0\right)$.

Using arguments similar to those used in Theorem 1 a) we can conclude that given $x \in \mathbb{Q}_{+}$and $i \in N$,

$$
f^{L}\left(x 1_{\{i\}}, 0\right)=x f^{L}\left(1_{\{i\}}, 0\right)
$$

Let $x$ be a non-negative irrational number. Then, there exists $\left(x^{l}\right)_{l \in \mathbb{N}}$ such that $x^{l} \in \mathbb{Q}_{+}$, $0<x^{l}<x$, and $\lim _{l \rightarrow \infty} x^{l}=x$. Thus, for all $i \in N$ and $l \in \mathbb{N}$,

$$
f^{L}\left(\left(x-x^{l}\right) 1_{\{i\}}, 0\right)=f^{L}\left(x 1_{\{i\}}, 0\right)-x^{l} f^{L}\left(1_{\{i\}}, 0\right) .
$$

Since $f^{L}\left(\left(x-x^{l}\right) 1_{\{i\}}, 0\right) \leq\left(x-x^{l}\right) 1_{\{i\}}$ and $\sum_{j \in N} f_{j}^{L}\left(\left(x-x^{l}\right) 1_{\{i\}}, 0\right)=0$, for all $i \in N$ and $l \in \mathbb{N}$,

$$
f^{L}\left(\left(x-x^{l}\right) 1_{\{i\}}, 0\right) \geq-\left(x-x^{l}\right) 1_{N}
$$


Thus, for all $i \in N$,

$$
-\left(x-x^{l}\right) 1_{N} \leq f^{L}\left(\left(x-x^{l}\right) 1_{\{i\}}, 0\right) \leq\left(x-x^{l}\right) 1_{\{i\}} .
$$

Then,

$$
0_{N}=\lim _{l \rightarrow \infty} f^{L}\left(\left(x-x^{l}\right) 1_{\{i\}}, 0\right)=f^{L}\left(x 1_{\{i\}}, 0\right)-x f^{L}\left(1_{\{i\}}, 0\right) .
$$

Since $f^{L}(c, E) \leq c$ and $\sum_{i \in N} f_{i}^{L}(c, E)=E$ for all $(c, E) \in \mathcal{L}$, we conclude that for all $i \in N, f^{L}\left(x 1_{\{i\}}, x\right)=x 1_{\{i\}}$, and $f^{L}\left(1_{\{i\}}, 0\right)=1_{\{i\}}-y^{i}$, where $y^{i} \in \mathbb{R}_{+}^{N}$.

We now prove that $y^{i}=y^{j}$ for all $i, j \in N, i \neq j$. Since $f^{L}$ satisfies $A 2$,

$$
\begin{aligned}
& f^{L}\left(1_{\{i, j\}}, 1\right)=f^{L}\left(1_{\{i\}}, 1\right)+f^{L}\left(1_{\{j\}}, 0\right)=1_{\{i\}}+1_{\{j\}}-y^{j} \\
& f^{L}\left(1_{\{i, j\}}, 1\right)=f^{L}\left(1_{\{i\}}, 0\right)+f^{L}\left(1_{\{j\}}, 1\right)=1_{\{i\}}-y^{i}+1_{\{j\}} .
\end{aligned}
$$

Then, $y^{i}=y^{j}$.

Given $i \in N$ we define $\omega=y^{i}$. It is trivial to see that $\omega$ is a weight system.

We now prove that $f^{L}=W R^{\omega}$. Given $(c, E) \in \mathcal{L}$, we consider a partition $\left\{N_{1},\{i\}, N_{2}\right\}$ of $N$ such that $c_{i}=c_{i}^{1}+c_{i}^{2}, c_{i}^{1} \geq 0, c_{i}^{2} \geq 0$, and $E=\sum_{j \in N_{1}} c_{j}+c_{i}^{1}$. Since $f^{L}$ satisfies $A 2$,

$$
\begin{aligned}
f^{L}(c, E) & =\sum_{j \in N_{1}} f^{L}\left(c_{j} 1_{\{j\}}, c_{j}\right)+f^{L}\left(c_{i}^{1} 1_{\{i\}}, c_{i}^{1}\right)+f^{L}\left(c_{i}^{2} 1_{\{i\}}, 0\right)+\sum_{j \in N_{2}} f^{L}\left(c_{j} 1_{\{j\}}, 0\right) \\
& =\sum_{j \in N_{1}} c_{j} 1_{\{j\}}+c_{i}^{1} 1_{\{i\}}+c_{i}^{2} f^{L}\left(1_{\{i\}}, 0\right)+\sum_{j \in N_{2}} c_{j} f^{L}\left(1_{\{j\}}, 0\right) \\
& =\sum_{j \in N_{1}} c_{j} 1_{\{j\}}+c_{i}^{1} 1_{\{i\}}+c_{i}^{2}\left(1_{\{i\}}-\omega\right)+\sum_{j \in N_{2}} c_{j}\left(1_{\{j\}}-\omega\right) .
\end{aligned}
$$

Now it is straightforward to prove that $f^{L}(c, E)=W R^{\omega}(c, E)$.

d) Suppose that $N=\{1,2\}, E=6$, and $c=(7,7)$. We can find $i \in N$ such that $f_{i}^{B}(c, E) \geq 3$. Assume without loss of generality that $i=1$.

Since $f$ satisfies $A 2, f_{1}^{B}(c, E)=f_{1}^{B}((6,1), 1)+f_{1}^{B}((1,6), 5) \leq 1+1=2$, which is a contradiction.

Remark 4. CONT is again needed in Theorem 2 a). We can use the same example as in Remark 3.

If we compare the rules satisfying $A 1$ (Theorem 1) with the rules satisfying $A 2$ (Theorem 2) in the four kinds of problems we obtain the following:

In $A P$ both classes of rules are unrelated. Moreover, an allocation rule $f^{A}$ satisfies $A 1, A 2$, and $C O N T$ if and only if for all $(c, E) \in \mathcal{A}, f^{A}(c, E)=E x$ where $x \in \mathbb{R}^{N}$ and $\sum_{i \in N} x_{i}=1$. 
In $S P$ the class of rules satisfying $A 2$ is a subset of the class of rules satisfying $A 1$ (just take $\omega(c)=\omega$ for all $c$ ).

In $L P$ the proportional rule, the only rule satisfying $A 1$, does not satisfy $A 2$. Moreover, the weighted-rights rules, which satisfy $A 2$, do not satisfy $A 1$.

In $B P$ the proportional rule, the only rule satisfying $A 1$, does not satisfy $A 2$.

\section{Characterizations of classical rules}

In this section we characterize the three classical rules based on the principles of "proportionality", "equal award", and "equal loss" using the additivity properties.

In Theorem 2 part c) we characterize the class of weighted-rights rules in $L P$. Notice that the weighted-rights rules also satisfy $A 2$ and $C O N T$ in $A P\left(\right.$ take $\beta_{i}(c)=c_{i}-\omega_{i} C$ and $x_{i}=\omega_{i}$ for all $\left.i \in N\right)$. Nevertheless, there are more allocation rules satisfying both properties. The next proposition characterizes the weighted-rights allocation rules as the only allocation rules satisfying $A 2$ and $C B$.

Proposition 1. An allocation rule $f^{A}$ satisfies $A 2$ and $C B$ if and only if $f^{A}$ is a weighted-rights rule $W R^{\omega}$ for some weight system $\omega$.

Proof. If $\omega$ is a weight system it is straightforward to prove that $W R^{w}$ satisfies $A 2$ and $C B$.

We now prove the converse. Let $f^{A}$ be an allocation rule satisfying both properties. We define $\omega$ as $f^{A}\left(0_{N}, 1\right)$. Since $f^{A}$ satisfies $C B$ we conclude that $\omega$ is a weight system.

Using arguments similar to those used in the proof of Theorem $1 \mathrm{~b}$ ) we conclude that for all $E \in \mathbb{R}_{+}, f^{A}\left(0_{N}, E\right)=E f^{A}\left(0_{N}, 1\right)=E \omega$.

Let $(c, E)$ be an allocation problem where $E \leq C$. As $f^{A}$ satisfies $C B$ we have that $f^{A}(c, C)=c$. Since $f^{A}$ satisfies $A 2$,

$$
\begin{aligned}
f^{A}(c, E) & =f^{A}(c, C)-f^{A}\left(0_{N}, C-E\right) \\
& =c-\omega(C-E) \\
& =W R^{\omega}(c, E) .
\end{aligned}
$$

The case $E>C$ is similar to the case $E \leq C$.

We now prove that this proposition is a tight characterization result.

$E S$ satisfies $A 2$ but not $C B$. 
Given $(c, E) \in \mathcal{A}$ and $i \in N$, we define the allocation rule $\psi$ as

$$
\psi_{i}(c, E)= \begin{cases}\min \left\{x, c_{i}\right\} & \text { if } C \geq E \\ R E_{i}(c, E) & \text { if } C<E\end{cases}
$$

where $\sum_{i \in N} \min \left\{x, c_{i}\right\}=E$. $\psi$ satisfies $C B$ but not $A 2$.

In Theorem 1, parts c) and d), we characterize the proportional rule in $L P$ and $B P$ as the only rule satisfying $A 1$. Nevertheless, in $A P$ and in $S P$ there are more rules satisfying these two properties. The next corollary shows that if we add $C O M$ to $A 1$ and $C O N T$ $(A 1)$, the proportional rule becomes the only rule satisfying these properties in $A P(S P)$. In this corollary we assume that $C>\mathrm{C}^{3}$.

Corollary 1. a) The proportional rule is the only allocation rule satisfying $A 1$, $C O N T$ and $C O M$.

b) The proportional rule is the only surplus rule satisfying $A 1$ and $C O M$.

Proof. a) Of course the proportional rule satisfies $A 1, C O N T$ and $C O M$.

Let $f^{A}$ be an allocation rule satisfying these properties. It is trivial to see that Theorem $1 a$ ) is also true if we restrict to the case $C>0$. Then, we conclude that for any $(c, E) \in \mathcal{A}, f^{A}(c, E)=E \alpha(c)$ where $\sum_{i \in N} \alpha_{i}(c)=1$. Since $f^{A}$ satisfies $C O M, f^{A}(c, C)=c$. Now it is easy to conclude that $\alpha(c)=\frac{c}{C}$, and hence, $f^{A}(c, E)=\frac{E}{C} c=P(c, E)$.

b) It is similar to the proof of part a).

Corollary $1 \mathrm{a}$ ) is a tight characterization result. RE satisfies CONT and COM but not $A 1$. Because of Remark 3 there exist rules satisfying $A 1$ and $C O M$ but not $C O N T$. We take the same example but with the function $\gamma$ defined as follows:

$$
\gamma(c, l)= \begin{cases}c \frac{B^{l}}{C} & \text { if } C^{\prime} s B^{l} \text {-th coordinate is non-zero } \\ 1_{N} \frac{B^{l}}{n} & \text { otherwise. }\end{cases}
$$

Finally, ES satisfies $A 1$ and $C O N T$ but not $C O M$.

Corollary $1 b)$ is a tight characterization result. $E S$ satisfies $A 1$ but not $C O M$. The surplus rule $\varphi$ defined as $\varphi_{i}(c, E)=\max \left\{0, c_{i}+\beta\right\}$ where $\sum_{i \in N} \varphi_{i}(c, E)=E$ satisfies $C O M$ but not $A 1$.

Moulin (1987) characterizes in his Theorem 2 the proportional surplus rule using $A 1$ and other properties. These properties are completely different from $C O M$ used in part b) of Corollary 1.

\footnotetext{
${ }^{3}$ In the general case $(C \geq 0)$ we will obtain that there exist many rules satisfying these properties. Nevertheless, all of them satisfy $f(c, E)=\frac{E}{C} c$ when $C>0$.
} 
Part a) of Corollary 1 is closely related to Theorem 5 in Chun (1988) where he proved that the proportional rule is the only allocation rule satisfying $A 1, C O N T^{*}$, and $C O M M^{4}$, In our result we change $C O N T^{*}$ to $C O N T$ (CONT is weaker than $\left.C O N T^{*}\right)$. Then, Chun's characterization of the proportional rule is also true only with continuity on the estate.

In Theorem 2 parts b) and c) and in Proposition 1 we characterize the class of weighted-sharing rules and weighted-rights rules in $A P, S P$, and $L P$ using $A 2$ and other properties. Unfortunately, in $B P$ there are no rules satisfying $A 2$. The next corollary shows that if we add $S Y M$ to the properties used in Theorem 2 and Proposition 1 we can characterize the equal-sharing rule in $S P$ and the rights-egalitarian rule in $A P$ and $L P$.

Corollary 2. a) The rights-egalitarian rule is the only allocation rule satisfying $A 2$, $C B$, and $S Y M$.

b) The equal-sharing rule is the only surplus rule satisfying $A 2$ and $S Y M$.

c) The rights-egalitarian rule is the only loss rule satisfying $A 2$ and $S Y M$.

Proof. It is similar to the proof of Corollary 1.

It is not difficult to check that Corollary 2 is a tight characterization result.

Since $C B$ implies $C O M$ and $R E$ satisfies $C B$, part a) of Corollary 2 can be obtained also as a consequence of Proposition 3 in Bergantiños and Méndez-Naya (2001), where they proved that $R E$ is the only allocation rule satisfying $A 2, C O M$, and $S Y M$.

Moulin (1987) characterizes in his Theorem 2 the equal-sharing surplus rule using several properties, which are different from the properties used in part b) of Corollary 2 . Nevertheless, Moulin (1987) and we use an additivity property, Moulin uses $A 1$ and we use $A 2$.

\section{Concluding Remarks}

The results obtained in Section 3 can be summarized in the following Table:

(Insert Table 1 here)

\footnotetext{
${ }^{4}$ Compatibility is called exact clearance in Chun (1988).
} 
The results obtained in Section 4 can be summarized in the following Table:

\section{(Insert Table 2 here)}

With $A 1$ and other properties we characterize the proportional rule in each of the four problems. With $A 2$ and other properties we characterize the "egalitarian" rules, the equal-sharing rule in surplus problems and the rights-egalitarian rule in loss and allocation problems. This suggests that additivity properties support the use of rules based on the three classical principles: $A 1$ supports the principle of "proportionality", and $A 2$ supports the principles of "equal award" and "equal loss".

\section{References}

Aczél, J., Dhombres, J., 1989. Functional equations in several variables. Encyclopedia of Mathematics and its Applications. Edited by G.C. Rota. Volume 31. Cambridge University Press.

Aumann, R.J., Maschler, M., 1985. Game theoretic analysis of a bankruptcy problem from the Talmud. Journal of Economy Theory 36, 195-213.

Bergantiños, G., Méndez-Naya, L., 2001. Additivity in bankruptcy problems and in allocation problems. Spanish Economic Review 3, 223-229.

Chun, Y., 1988. The proportional solution for rights problems. Mathematical Social Sciences 15, 231-246.

Herrero, C., Maschler, M., Villar, A., 1999. Individual rights and collective responsibility: the rights-egalitarian solution. Mathematical Social Sciences 37, 59-77.

De Frutos, M.A., 1999. Coalitional manipulation in a bankruptcy problem. Review of Economic Design 4, 255-272.

Moulin, H., 1987. Equal or proportional division of a surplus, and other methods. International Journal of Game Theory 16, 161-186.

Moulin, H., 2000. Priority rules and other inequitable rationing methods. Econometrica $68,643-684$.

Moulin, H., 2002. Axiomatic cost and surplus sharing. In Arrow, K., Sen, A., Suzumura, K. (Eds) Handbook of Social Choice and Welfare. Elsevier, Amsterdam, pp. 289-357.

O’Neill, B., 1982. A problem of rights arbitration from the Talmud. Mathematical Social Sciences 2, 345-371.

Thomson, W., 2003. Axiomatic and game-theoretic analysis of bankruptcy and taxation problems: a survey. Mathematical Social Sciences 45, 249-297. 
Young, P., 1988. Distributive justice in taxation. Journal of Economic Theory 43, 321335. 
Table 1

\begin{tabular}{|l|c|c|}
\hline \multicolumn{3}{|c|}{ Additive rules } \\
\hline Allocation & and $C O N T$ & $A 2$ \\
& $E \alpha(c)$ & $\begin{array}{c}\text { and } C O N T \\
\beta(c)+E x\end{array}$ \\
\hline Surplus & $E w(c)$ & Weighted-sharing \\
& $w(c)$ is a weight system & \\
\hline Loss & Proportional & Weighted-rights \\
\hline Bankruptcy & Proportional & \\
\hline
\end{tabular}


Table 2

\begin{tabular}{|l|c|c|}
\hline \multirow{3}{*}{ Characterizations of classical rules } \\
\hline Surplocation & Proportional with & Rights-egalitarian with \\
& $A 1, C O N T$, and $C O M$ & $A 2, C B$, and $S Y M$ \\
\hline Loss & Proportional with & Equal-sharing with \\
& $A 1$ and $C O M$ & $A 2$ and $S Y M$ \\
\hline Bankruptcy & Proportional with & Rights-egalitarian with \\
& $A 1$ & $A 2$ and $S Y M$ \\
\hline
\end{tabular}

